\title{
New Transmission for Electric and Hybrid Vehicles
}

\author{
R. G. Khadeev \\ State Research and Test Institute of Military Medicine, Moscow, Russia \\ Email: khadeev@mail.ru
}

How to cite this paper: Khadeev, R.G. (2020) New Transmission for Electric and Hybrid Vehicles. Engineering, 12, 229-233. https://doi.org/10.4236/eng.2020.124018

Received: March 2, 2020

Accepted: March 29, 2020

Published: April 1, 2020

Copyright $\odot 2020$ by author(s) and Scientific Research Publishing Inc. This work is licensed under the Creative Commons Attribution International License (CC BY 4.0).

http://creativecommons.org/licenses/by/4.0/

\begin{abstract}
The present article is concerned with a method for automatic control of the transmission ratio and torque moment from the engine to the driven wheel and transmission construction in the way that the engine can operate during the entire time of the vehicle's movement at the optimum condition. It is proposed to consider a new vehicle transmission device. Usually every innovation goes through three stages of consideration: 1) It is a rubbish. 2) There is something in it. 3) That is how it should be, what is complicated in it, it's very simple. The proposal project is now in the first stage with the transition to the second stage. However, the transmission mechanism is simple, it works well and there is hope for its future prospects.
\end{abstract}

\section{Keywords}

Epicyclical Differential, Infinitely Variable Transmission, Simple Electrical Control

\section{Introduction}

We can facilitate and reduce the price of the vehicle transmission by applying the design, proposed in this article. The transmission contains mechanisms, placed in series, consists of an epicyclical asymmetric differential gear, which is capable of rotating around an axis, and connected to the high power-sliding coupling. This infinitely variable transmission allows the engine to operate at optimal condition during run up and most of the operating modes and has a simple electrical control without mechanical switches. It is acknowledged that it is necessary to make a smooth change of the transmission ratio within limits of 25 - 30 to work at optimal engine running. 


\section{Method}

It contains series-connected mechanisms consisting of a planetary differential connected to a generator, which has a stator that can rotate about the axis, forming together an electric machine of double rotation. One of such mechanisms consisting of a generator connected to an asymmetrical planetary differential is shown on the scheme, Figure 1.

\section{Operation of the Device}

The proposed design makes it easy to achieve this task. The transmission diagram is shown in Figure 1.

The first mechanism consists of a generator and an asymmetric differential. The differential input, the differential gear 7 and the generator rotor 2 are connected to the drive shaft 1 . One output of the differential, the crown of the differential 5 is connected to the stator of the generator 3 , which has the ability to rotate around the shaft, forming a double-rotation electric machine with the rotor. The generator stator is also connected to the coupling 4, which, if necessary, fixes it by connecting it to the housing. The second output, the pinion cage of the planetary differential with pinion gears 6 , is connected to the input of the second mechanism, consisting of a planetary differential connected to the power sliding coupling 8 . The differential input, the differential gear 14, transmits rotation to the pinion cage 13 and the crown 11 . Its output, the pinion cage 13 , is connected to the mating part of the power sliding coupling 8 , and is also connected with the coupling 10, which, if necessary, fixes it, connecting it to the housing. The second output of the planetary differential, the crown 11 is connected to the input

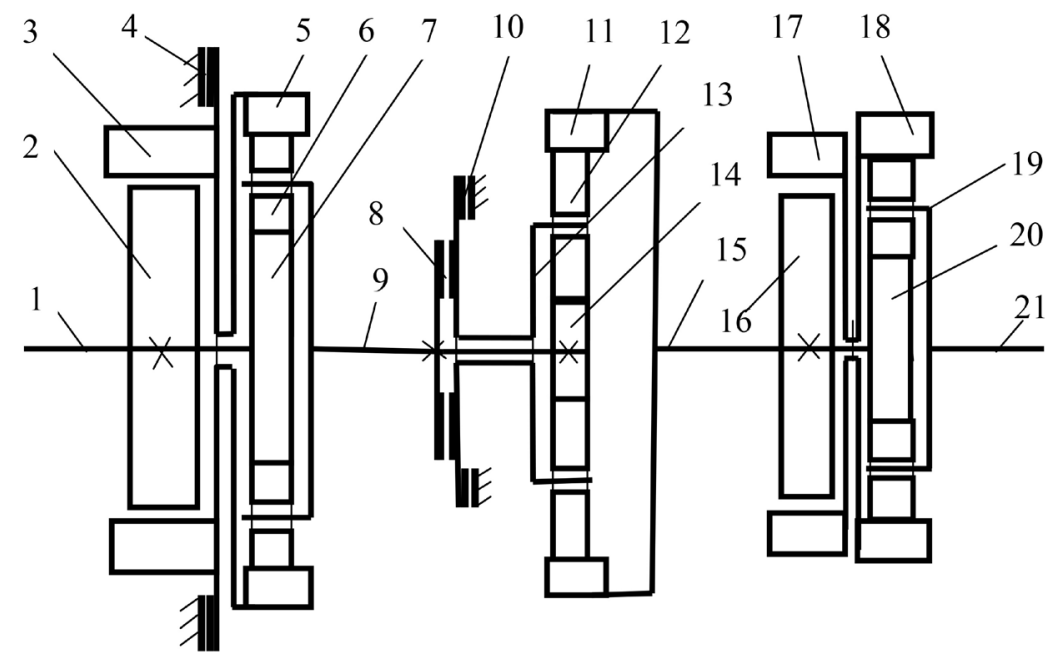

Figure 1. Scheme of the method. 1. Motor shaft. 2. Generator rotor. 3. Generator stator. 4. Coupling, connecting the stator to the housing. 5. Crown of the planetary differential. 6. Pinion gears. 7. Differential gear. 8. Solid coupling. 9. Intermediate shaft. 10. Coupling connecting the pinion cage to the housing. 11. Crown. 12. Pinion gears. 13. Pinion cage. 14. Differential gear. 15. Intermediate shaft. 16. Armature of the eddy-current clutch. 17. Inductor of the eddy-current clutch. 18. Crown of the planetary differential. 19. Pinion cage. 20. Differential gear. 21. Driven shaft. 
of the third mechanism, consisting of a planetary differential connected to an eddy current clutch. The input of the differential is connected to the eddy current clutch inductor 16 and the gear wheel of the differential gear 20. A single output is connected to the crown 18 and to the anchor of eddy current clutch 17 and the second output of the differential, pinion cage 19 , is connected with the driven shaft of the transmission 21.

When the drive shaft 1 rotates, the generator, first mechanism generates a current and the stator 3 with the rotor 2 act the part of a power sliding coupling, since in the presence of an electric load in the generator circuit, a force arises between it, dragging the stator behind the rotor, partially blocking the operation of the differential, forcing the mechanism to rotate around the axis. In this case, part of the rotation is transmitted through the differential gears, and part of the rotation of the mechanism around the axis. When the electric load current increases in the generator circuit, the gripping power between the stator and the rotor decreases, the slip between them decreases, the cumulative transmission ratio from the drive shaft to the device output decreases, and the driven shaft accelerates. When the load on the drive shaft increases, it slows down; the rotation from the engine is more transmitted through the differential gears, and the gear ratio of the mechanism increases. The torque on its driven shaft depends on the transmission ratio of the planetary differential. When the drag moment increases, when the shaft is braked and the slip between the stator and the rotor increases, more rotation is transmitted through the differential gears, the overall gear ratio increases, and the torque at its output increases. In addition, when the slip between the stator and the rotor increases, the ampere force increases and the torque increases more. You can also control the amount of sliding in the generator, which means the transmission ratio and the torque of the mechanism, by changing the electrical load in the generator circuit. Generator stator 3 is connected to the coupling 4 with a synchronizer to stop it, connecting it to the housing. This makes it possible to use the generator for accumulating energy in batteries, as well as when using it, if necessary, as an electric motor for starting.

From the output of the first mechanism, the rotation goes to the differential gear 14 , and connects to the coupling 8 . One output of the second mechanism, the crown 11, is connected to its output shaft, and the second output, the pinion cage 12 , is connected to the mate of the power sliding coupling 8 . This mechanism performs the function of the coupling mechanism, and increases the torque at its output during its operation, when there is a slip in the clutch, mainly at the start. After the coupling is fully connected, when there is no slipping motion, the torque at the output of the mechanism is equal to the torque at its input, and the transmission ratio is equal to one. The second output of the differential, the pinion cage 12 , can also be connected to the coupling 10 fixing it to the housing, which leads to the reverse direction of rotation of the output shaft, including the reverse speed. From the output of the second mechanism, the rotation is fed to the input of the differential, connected to the eddy current clutch, to the solar gear 20, and connects to the anchor of the eddy current clutch 16 . One output of 
the differential, the pinion cage 19, is connected to the driven shaft of the transmission 21 , and the second output, the crown 18, is connected to the inductor of the eddy-current clutch 17 . When the transmission shaft is loaded, a sliding motion occurs between the inductor and the armature of the eddy current clutch, and part of the rotation will be transmitted through the differential elements. The transmission ratio and torque will increase. As the load increases, the sliding motion will increase, this will lead to an increase in ampere strength and an increase in torque. By changing the exciting current of the eddy current clutch, you can also control the torque on the drive shaft. In this mechanism, a second generator can be installed instead of an eddy current clutch.

The electric current generated by the generator can be used to the driving gear of wheels, that are not connected to the engine, as well as, for example, to produce hydrogen, to supply it with gasoline vapors to the cylinders, to charge batteries, or for other purposes. For driving wheels that are not connected to the engine, it is possible to connect an electric motor to them through a similar mechanism consisting of a planetary differential and an eddy current clutch, which allows you to automatically change the transmission ratio and torque on the wheel, depending on the required value, at constant and optimal engine running. Its device is described in detail in [1].

Such a transmission automatically, in accordance with the power of the engine now, comparing it by means of a differential with the power needed to overcome the electric induction force, sets the gear ratio to the driven shaft. Losses in such manner of mounting are insignificant. Typically, losses in the eddy current clutch are $0.7 \%-3 \%$, and in nominal modes do not exceed $1 \%$. Mechanical losses in differentials are also low. Both differential arms rotate the driven shaft in one direction, summing up the force of both outputs. This transmission is the most convenient for electric and hybrid vehicles. In a simplified version, it can maintain optimal speeds by means of a regulator that controls the existing current in the eddy current clutch and the generator load current in accordance with the engine power at the moment, increasing the current when the engine power increases and decreasing current when the engine power decreases. The transmission can be simplified in some cases, depending on the properties required for a particular vehicle. You can exclude the coupling mechanism with the inclusion of the reverse speed. Alternatively, instead of the coupling mechanism, include in the scheme a coupling with a synchronizer. Then electric traction can be used to reverse the direction of travel, as shown in [2]. A third mechanism that includes an electro-induction clutch and differential can be excluded, and an eddy current clutch that works in parallel with the generator can be included in the first mechanism. The diagram of such a device is shown in Figure 2.

In this case, in the same mechanism there are two different pairs of associated by mutual induction elements involved in the transformation of transmission ratio, in a single regulator, a generator, and eddy current clutch, in this case the current, generated by the generator is spent on the inductor of eddy current 


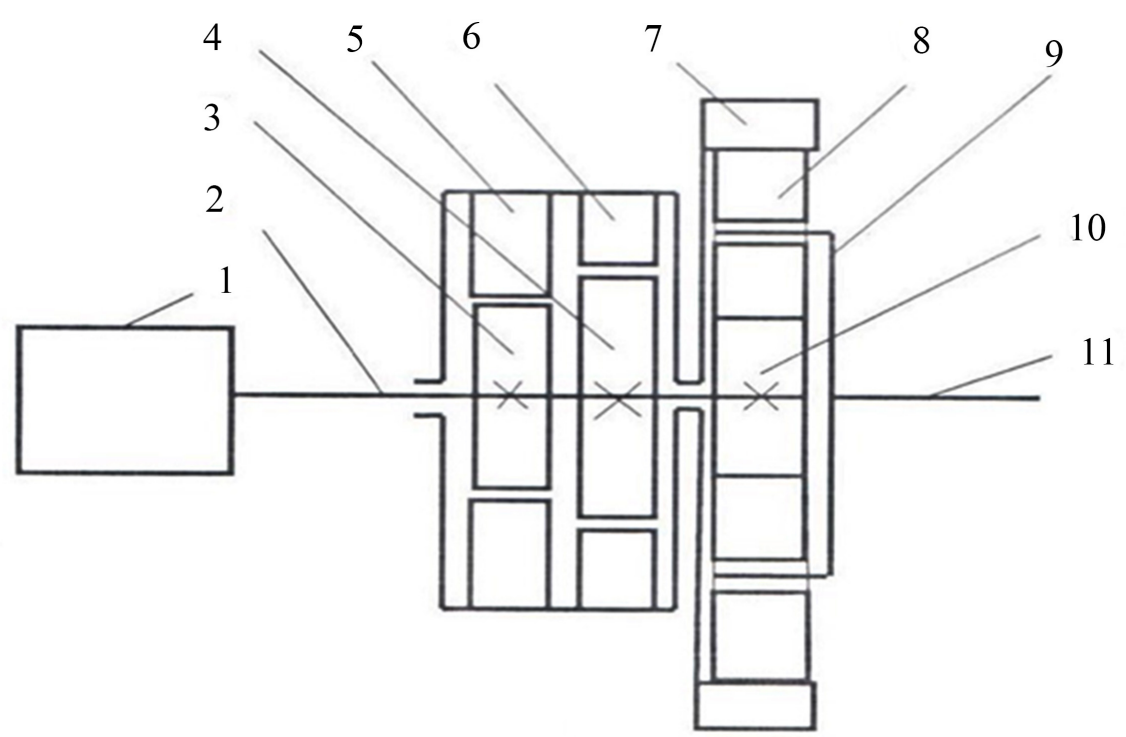

Figure 2. Engine. 2. Drive shaft. 3. Generator rotor. 4. Inductor of the eddy-current clutch. 5. Generator stator. 6. Coupling anchor. 7. Crown of the planetary differential. 8. Pinion gears. 9. Differential pinion cage. 10. Differential gear. 11. Output shaft.

clutch. In addition, if the driving gear is electric, the generator can provide current to the motor rotor existing winding. In this case, neither the electric motor nor the eddy current clutch will need current collectors with running contacts, because the generator rotor, the motor rotor, and the inductor of the electric induction clutch are located on a common shaft. This will increase the reliability of the drive and increase its service life. Control of existing currents can be performed by installing a radio-electronic device on the shaft, which also receives power from the generator. For example, you can use the Hall sensor to do it. The main advantages of the proposed design:

1) Operation of the engine at the calculated economy modes during almost the entire driving cycle. It significantly increases efficiency.

2) No mechanical switching in the entire operating range. This results lead to reliability and durability.

3) Relative simplicity of design and low cost.

\section{Conflicts of Interest}

The author declares no conflicts of interest regarding the publication of this paper.

\section{References}

[1] Khadeev, R.G. (2018) The New Transmission for a Hybrid Car. Engineering, 10, 125-129. https://doi.org/10.4236/eng.2018.104009

[2] Khadeev, R.G. (2019) New Transmission for a Hybrid. Journal of Multidisciplinary Engineering Science and Technology, 6, 10523-10525. 